\title{
Characteristics and Trends of English Testing Research in China: Visual Analysis Based on CiteSpace
}

\author{
Na Ming \\ China West Normal University, Nanchong, Sichuan, China
}

\begin{abstract}
This thesis aims to have a visualized analysis of English testing from 1995 to 2020 in China with the help of the Scientometric software CiteSpace by drawing a map of keyword co-occurrence, time zone, and author cooperation network as well as scientific research institution cooperation network. It found that the cooperation between the authors is not strong and so is the research institutions. Besides, research focus detected mainly include college English, formative assessment, assessment, college English teaching, summative assessment and language testing. And according to the map of time zone, this research field is constantly moving towards enrichment and refinement. Finally, based on the mapping knowledge domains drawn by CiteSpace, the future development trends and deficiencies of this research are summarized.
\end{abstract}

Key words: English testing; CiteSpace; research focus; visualized analysis

\section{Introduction}

In recent years, English testing activities have attracted much attention in foreign academic circles and have yielded fruitful achievements. However, systematic research on English testing in China lags behind, so it is valuable to learn about the status of English testing research, research hotspots, key points, main problems and the future study of English testing research in China, in order to promote the development of English testing and improve the effects of English teaching and learning. Therefore, the research uses CiteSpace5.7.R3 to conduct statistics and analysis of English test research papers from CSSCI.

\section{Methods}

This study intends to answer the following questions: (1) What are the hot spots and key points of English testing research in China? (2) Which research teams are engaged, and what is the current research status? (3) What are the main problems or bottlenecks? (4) What is the future direction?

"English testing" or "English evaluation" are decided as the subject without limiting publication time to be retrieved. The language is set to Chinese, and all the papers retrieved are from CSSCI in the China National Knowledge Infrastructure (CNKI). A total of 477 articles were retrieved. In order to ensure the accuracy of the literature, the literature that is extremely low or irrelevant to the English test was manually eliminated, and finally 470 search results were obtained as the research object.

The data was last updated on February 26, 2021. This article uses 1 year as time slicing, and divides the time from 
1998 to 2021 into 24 time slices. Literature are exported by using the Refworks in the CNKI, and then the data are imported into CiteSpace5.3R. And the data are converted using the data function of CiteSpace. After creating a new project, keywords, authors and institutions are selected as the node types respectively, and CiteSpace was run to generate a keyword co-occurrence knowledge map, time zone view, author and institutional cooperation map.

\section{Results and Analysis}

3.1 The overall development of English testing research in China

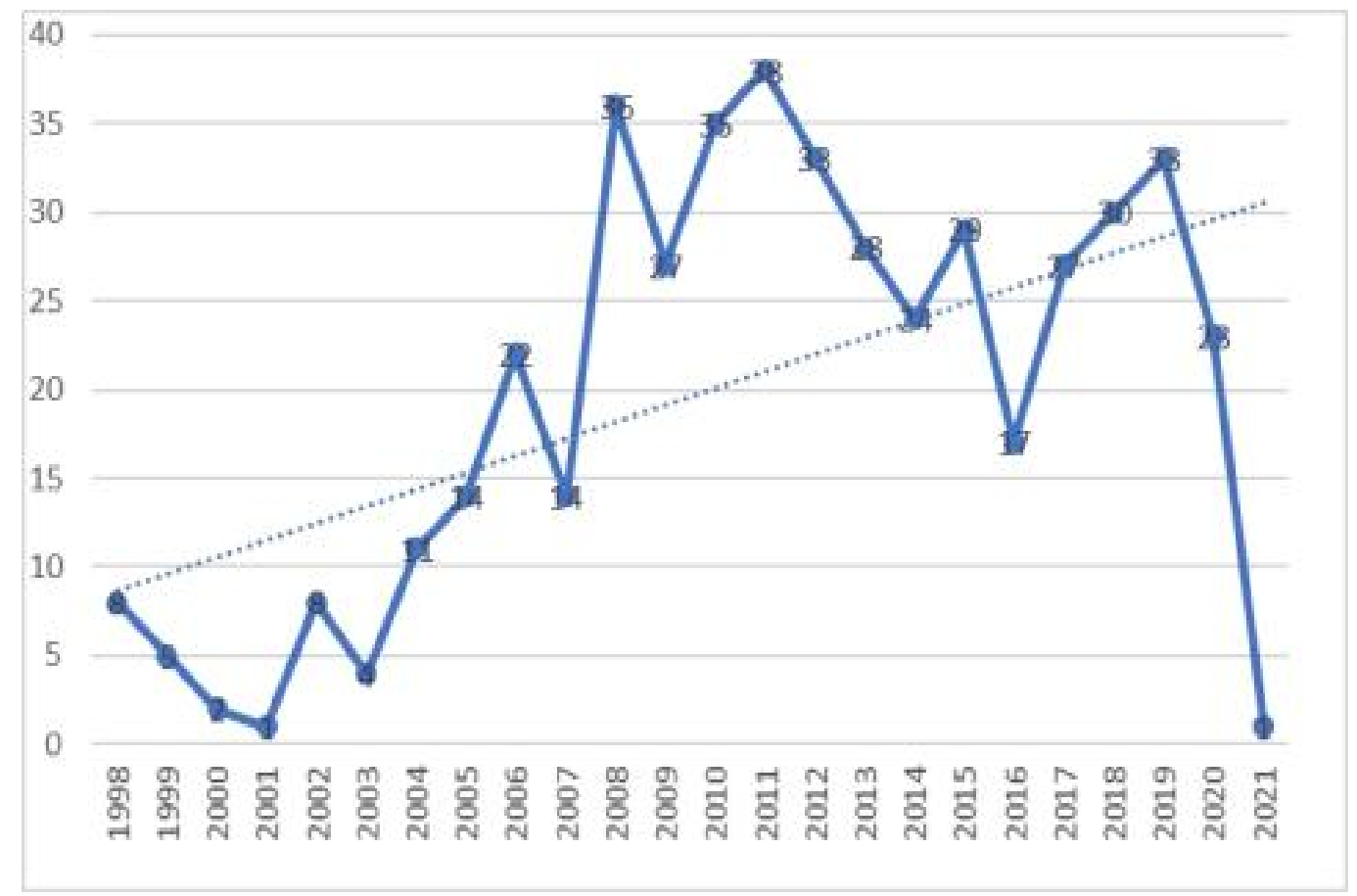

Figure 1. Annual publication

From Figure 1, the embryonic development period of English testing research was from 1998 to 2003, and the number of related articles published every year was less than 10. From 2004 to 2007, the development of English testing research was on the rise. The number of related articles published each year exceeded 10, and in 2006, it even reached 22. From 2008 to now, the period of development of English testing research is rapid, and the average number of related articles published every year is about 30. Although the amount publication of English tests in the country has dropped slightly during the development period, the number has shown an overall upward trend over the past two decades, indicating that English tests have gradually attracted the attention of academic circles in China. This result is consistent with the current research status in academia. Jiang studied articles on English testing research published in eight major foreign language core journals in China from 1996 to 2005, and found that in the past 10 years, testing research has generally shown an upward trend.

\subsection{Analysis of keyword co-occurrence knowledge map}

Keywords are the condensing and refining of the core content of the article. If a keyword appears repeatedly in the literature of its field, the research topic represented by the keyword is a research hotspot. The keyword co-occurrence analysis time is from 1998 to 2021, and the time slice is one year, which is divided into 24 time slices. Node types are key words, and top50 is selected for each time slice. Cosine is selected for connection strength, and the network is cut using MST (Minimum Spanning Tree) algorithm to simplify the network. After running, we obtained a keyword co-occurrence knowledge map with 67 nodes, 43 connections, and 0.0194 density, as shown in Figure 2. At the same time, a list of keyword frequency and centrality data were obtained in Table 1. 
Table 1. Keyword frequency and centrality

\begin{tabular}{cccc}
\hline Number & Keyword & Frequency & Centrality \\
\hline 1 & College English & 46 & 0.30 \\
2 & Formative Evaluation & 39 & 0.19 \\
3 & Evaluation & 20 & 0.01 \\
4 & College English Teaching & 0.00 \\
5 & Summative Evaluation & 8 & 0.03 \\
6 & Language Test & 8 & 0.19 \\
7 & English Teaching & 8 & 0.05 \\
8 & Backwash & 7 & 0.01 \\
9 & Teaching Evaluation & 6 & 0.00 \\
10 & English Test & 6 & 0.05 \\
11 & Core Literacy & 6 & 0.00 \\
12 & Reliability & 5 & 0.00 \\
13 & Oral Test & 4 & 0.00 \\
14 & Foreign Language Teaching & 4 & 0.14 \\
15 & Content Validity & 4 & 0.00 \\
16 & Validity & 4 & 0.00 \\
17 & Self-learning & 4 & 0.00 \\
18 & Evaluation System & 4 & 0.00 \\
19 & Self-evaluation & 4 & 0.00 \\
\hline 0 & & 3 & 0.00 \\
\hline
\end{tabular}

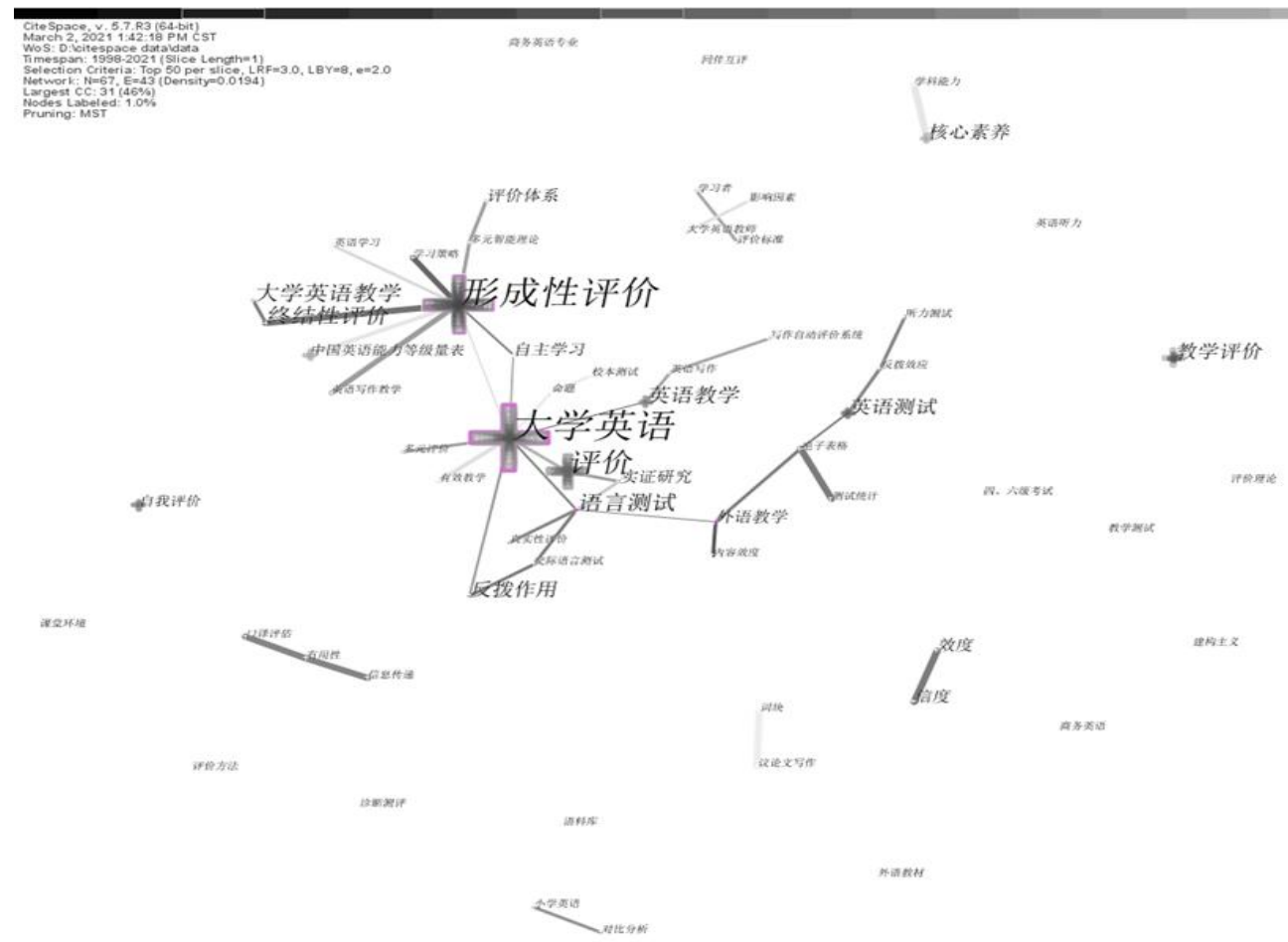

Figure 2. Keyword co-occurrence knowledge map 
From Figure 2, we can know that the node of "College English" is the largest, followed by "Formative Evaluation", "Evaluation", "College English Teaching", "Summative Evaluation", "Language Test", "English Teaching", and "Backwash". Further analysis of the high-frequency keywords and centrality lists shown in Table 2 shows that there are only three high-frequency words with more than ten, which are "University English", "Formative Evaluation" and "Evaluation". From the perspective of centrality, there are nine keywords whose centrality exceeds 0.1 , which are "University English", "Formative Evaluation", "Evaluation", "Summative Evaluation", "Language Tes", "English Teaching", "Backwash", "English Test" and "Foreign Language Teaching". Some keywords have a high frequency and centrality, and show a positive correlation, such as "college English" and "formative evaluation". Formative evaluation is an important means of college English teaching evaluation, tracking, monitoring and feedback on the teaching and learning process, which shows that the use of formative evaluation methods to evaluate the effects of college English teaching and learning is not only a foreign study but also the focus and hotspot in China. Yang and Liu elaborated the background of the implementation of formative evaluation in college English teaching, and then stated the college English teaching based on formative evaluation from the aspects of curriculum currency, credit bank, formative evaluation teaching practice, development of formative evaluation teaching platform, and establishment of electronic portfolio. It is worth noting that the frequency of "Foreign Language Teaching" is not high, but it also plays a pivotal role in related research, and it is also a hot topic discussed in this field.

3.3 Analysis of knowledge map of authors and institutions

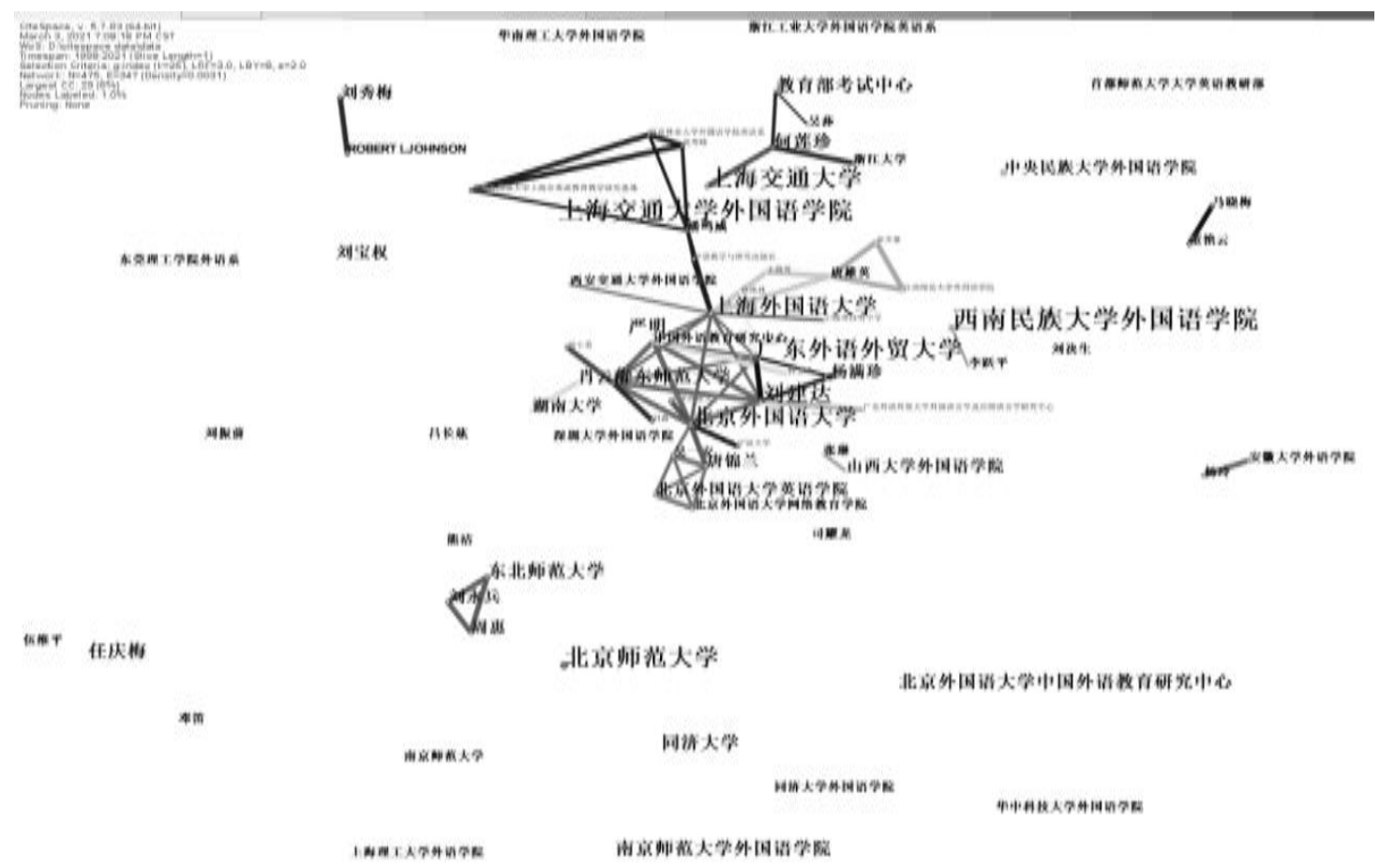

Figure 3. Knowledge map of authors and institutions

Scientific cooperation means that research scholars work together for the common purpose of producing new scientific knowledge. "Author" and "institution" are selected for node types, and the time slice is one year from 1998 to 2021. Other parameters are set to the default settings.

From figure 3, there are not many highly productive authors and institutions, and the cooperation between authors needs to be strengthened, and there are not many groups that form a cooperative scale. Jin Yan (6 articles) of Shanghai Jiaotong University (16 articles) and Liu Jianda (6 articles) of Guangdong University of Foreign Studies (13 articles) are the main highly productive authors in this field. Jin Yan, as one of the leading tasks of the current English testing research, 
mainly studies the subject of college English testing. In 2010, she explored the application of earning-promoting evaluation in college English teaching, expounding the importance of multi-evaluation with the purpose of promoting learning for experiential college English teaching. Based on China's Standards of English Language Ability, Liu Jianda not only introduced it, but also expounded its relationship with English learning, and focusing on comprehensive application, learner-centeredness and the principle of gradual and orderly progress, and he expounded the enlightenment of China's Standards of English Language Ability to English learning. In addition, Han Baocheng (3 articles) of Beijing Foreign Studies University (14 articles) discussed the evaluation of Chinese English teachers' professional competence and the framework of the English test objectives as well as content setting of the college entrance examination from teachers and students. Zou Shen (2 articles) of Shanghai International Studies University (13 articles), taking the writing test validity research as a starting point, briefly described the theory and practice of writing test validity research, and used corpus as an example to explore new ways of writing validity research. On the whole, English testing is an important part of teaching and learning, and its related research has not yet received enough attention from the academic community. Although the teams represented by Liu Jianda, Han Baocheng, Zou Shen, and Jin Yan have achieved certain development, still more attention should be paid from academia and investment are needed.

3.4 Analysis of time zone view

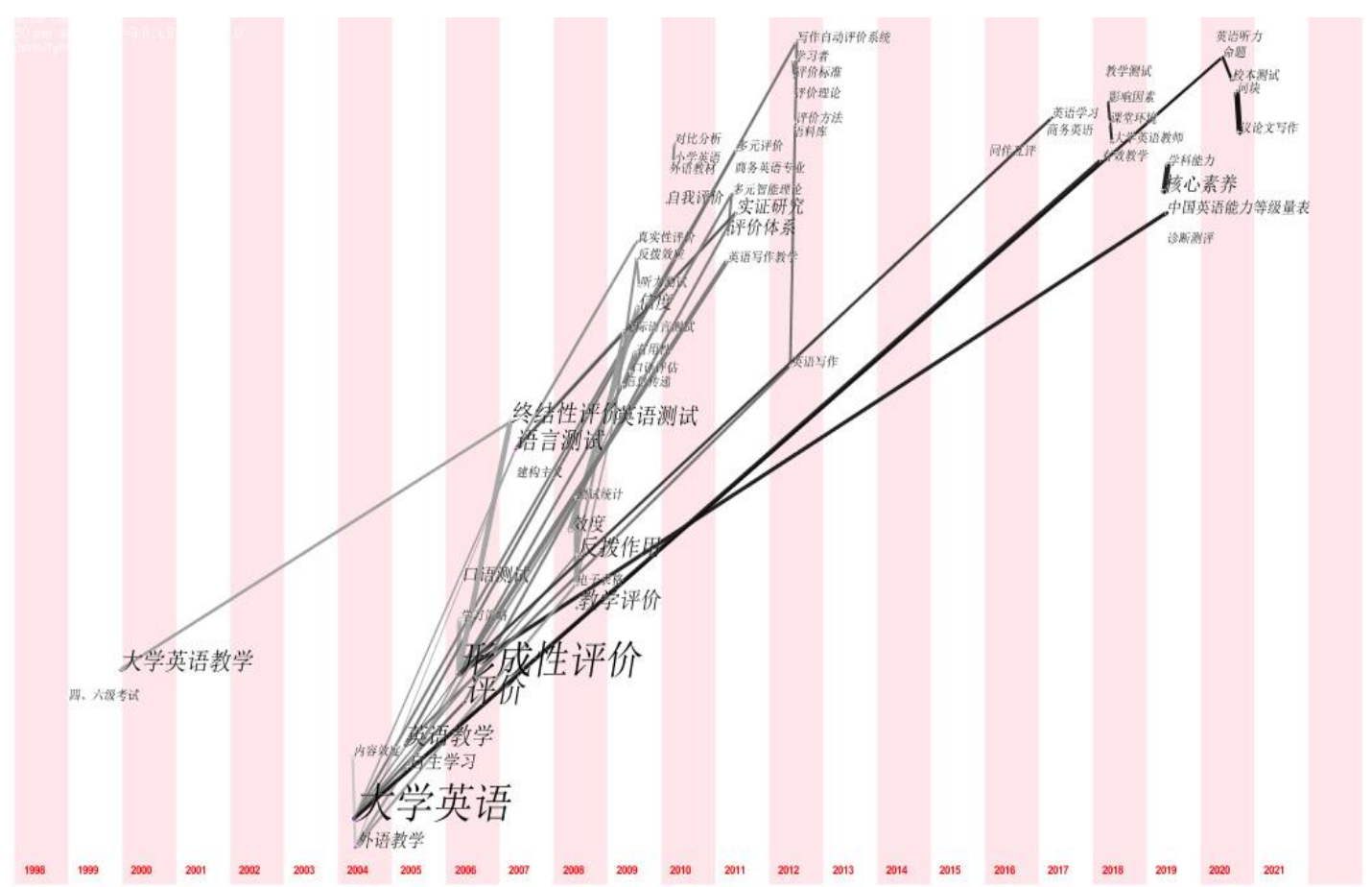

Figure 4. Time zone view

In order to further understand the research hotspots of English testing in different periods, the keyword time zone view as shown in Figure 4 was drawn through CiteSpace. Through the connection between the time periods, the inheritance between the time periods can be seen. By analyzing Figure 3, we can see that the early (1998-2003) research was mainly centered on CET-4 and CET-6. In the early days, the keywords "English Test" or "English Evaluation" did not appear. The period from 2004 to 2012 was a period of vigorous development, and many interrelated keywords appeared, such as "Content Validity", "Formative Evaluation", "Oral Test", "Summative Evaluation", and "Washback" related to English testing. The research objects are also getting younger and younger, gradually expanding from "college English" to "primary school English", "business English", "English teaching" and so on. At the same time, they began to pay more attention to keywords such as "self-learning", "self-evaluation" and "learner", indicating that the initiative of evaluation is 
gradually changing. From 2013 to 2015, English testing research entered a gestation period, and no keywords appeared at this time. Since 2016, the English test research has entered a new period of development. This period paid more attention to the development of English testing under the general background. The research perspective is no longer limited to simple tests, but considers the development of English testing from a social perspective, such as "Peer Evaluation", "Classroom Environment", and "School-based Testing".

\section{Conclusions}

Analyzing 470 CSSCI papers obtained from CNKI through CiteSpace, the following conclusions are obtained:

(1) At present, the focus and hot spots of English testing research in China are "College English", "Formative Evaluation", "Evaluation", "College English Teaching", "Summative Evaluation", and "Language Testing".

(2) The groups currently engaged in research in this field mainly include Jin Yan from Shanghai Jiaotong University, Liu Jianda from Guangdong University of Foreign Studies, Han Baocheng from Beijing Foreign Studies University, and Zou Shen from Shanghai International Studies University.

In the past 30 years, the English test has attracted the attention of the academic community in China. The research content has been further deepened and refined, and the number of research results has shown an upward trend as a whole. However, there are still deficiencies in English testing research. First of all, the scale of cooperation needs to be strengthened and improved. In addition, research hotspots and priorities are unbalanced. Through analysis of the retrieved literature, it is found that the "University English" and "Formative Evaluation" far surpass other keywords in terms of centrality and frequency, and they are undoubtedly the focus of research. But the research objects should be gradually enriched and specific. The evaluation method should also be transformed, and only a comprehensive evaluation method can be used to ensure the validity and authenticity of the evaluation.

\section{Conflicts of Interest}

The author declares no conflicts of interest regarding the publication of this paper.

\section{References}

[1] Jiang X.J. (2007). Domestic English Test Research: A Decade of Review and Prospect. Foreign Language World, (02):89-96.

[2] Feng G., Wang K.F, Liu X. (2014). The Mapping Analysis of Scientific Knowledge in the Recent Twenty Years of International Translation Studies. Technology Enhanced Foreign Language Education, (01):11-20.

[3] Jin Y. (2020). The Current Situation Investigation and Reform Direction of College English Evaluation and Testing. Foreign Language World, (05):2-9.

[4] Yang M.Z., Liu J.D. (2019). Practice of College English Teaching Based on Formative Assessment, Technology Enhanced Foreign Language Education, (03):97-102.

[5] Katz J. S., Martin B. R. (1997). What is Research Collaboration? Research Policy, 26(01):1-18.

[6] Jin Y. (2010). Multiple Evaluation of Experiential College English Teaching. Foreign Languages in China, $7(01): 68-76+111$.

[7] Liu J.D. (2017). Chinese English Competence Scale and English Learning. Foreign Languages in China, 14(06):4-11. 